\title{
The BeiDou Navigation Message
}

\author{
Oliver Montenbruck \\ DLR, German Space Operations Center, Germany \\ Phone +49(8153)28-1195, fax -1450, oliver.montenbruck@dlr.de \\ Peter Steigenberger \\ Technische Universität München, Germany \\ steigenberger@bv.tum.de
}

\begin{abstract}
The article provides an overview of the BeiDou navigation message contents and highlights its specific communalities and differences with respect to other GNSS constellations. Making use of data collected by multi-GNSS monitoring stations of the MGEX and CONGO networks, the quality of BeiDou broadcast ephemerides is assessed through the analysis of satellite laser ranging measurements, comparison with post-processed orbit and clock products as well as positioning tests. Specific attention is given to signalspecific group delays and their proper consideration in the positioning.
\end{abstract}

KEYWORDS: BeiDou, Broadcast Ephemeris, multi-GNSS positioning, TGD, MGEX

\section{INTRODUCTION}

Following the great success of the Global Positioning System (GPS) and the completion of the Russsian GLONASS system, BeiDou is the third navigation satellite system that offers an independent, though regional, positioning service. Up to the end of 2012, a total of 6 geostationary satellites (GEO), five satellites in inclined geosynchronous orbit (IGSO) and five satellites in medium altitude Earth orbits (MEO) with an orbital period of $12 \mathrm{~h} 53 \mathrm{~m}$ have been launched. Aside from a failed GEO satellite (G2) and the first MEO satellite (M1=C30) that is no longer fully operational (Hauschild et al. 2012a), the constellation comprises a total of 14 active satellites (Table 1). Given the placement of the GEO and IGSO satellites, the BeiDou system provides best coverage in the Asia-Pacific region. Users in this area can benefit from BeiDou as either a supplement to legacy navigation systems or as a stand-alone system. Other than for QZSS (Inaba et al. 2009), the orbital geometry and coverage of the BeiDou constellation is fully symmetric with respect to the equator. Nevertheless, users in the northern hemisphere (specifically China) benefit from a lower latency and improved quality of navigation information. 
Table 1. BeiDou constellation status as of April 2013 (see http://igs.org/mgex and CSNO 2012)

\begin{tabular}{llll}
\hline Satellite & Int. Sat. ID & PRN & Notes \\
\hline M1 & 2007-011A & C30 & Suspected clock problems (Hauschild et al. 2012a) \\
G2 & 2009-018A & n/a & Inactive; uncontrolled \\
G1 & 2010-001A & C01 & $140.0^{\circ} \mathrm{E}$ \\
G3 & 2010-024A & C03 & $110.5^{\circ}$ (moved from $84.0^{\circ}$ to new position between \\
G4 & 2010-057A & C04 & $160.0^{\circ} \mathrm{E}$ \\
I1 & $2010-036 \mathrm{~A}$ & C06 & $\sim 120^{\circ} \mathrm{E}$ \\
I2 & $2010-068 \mathrm{~A}$ & C07 & $\sim 120^{\circ} \mathrm{E}$ \\
I3 & $2011-013 \mathrm{~A}$ & $\mathrm{C} 08$ & $\sim 120^{\circ} \mathrm{E}$ \\
I4 & $2011-038 \mathrm{~A}$ & $\mathrm{C} 09$ & $\sim 95^{\circ} \mathrm{E}$ \\
I5 & $2011-073 \mathrm{~A}$ & $\mathrm{C} 10$ & $\sim 95^{\circ} \mathrm{E}$ \\
G5 & $2012-008 \mathrm{~A}$ & $\mathrm{C} 05$ & $58.75^{\circ} \mathrm{E}$ \\
M3 & $2012-018 \mathrm{~A}$ & $\mathrm{C} 11$ & \\
M4 & $2012-018 \mathrm{~B}$ & $\mathrm{C} 12$ & \\
M5 & $2012-050 \mathrm{~A}$ & $\mathrm{C} 13$ & \\
M6 & $2012-050 \mathrm{~B}$ & $\mathrm{C} 14$ & \\
G6 & $2012-059 \mathrm{~A}$ & $\mathrm{C} 02$ & $80.0^{\circ} \mathrm{E}$ \\
\hline
\end{tabular}

While signals have been continuously available throughout the deployment and testing of the BeiDou system, a public interface control document (ICD; CSNO 2012) has only been released in Dec. 2012 along with the announcement of a full regional navigation service.

All BeiDou satellites transmit signals on a total of three frequencies $(\mathrm{B} 1=1561.098 \mathrm{MHz}$, B2 $=1207.140 \mathrm{MHz}$, and B3=1268.520 MHz). Even though only the B1I open service codes are disclosed in the public ICD, the basic signal structure and code generators of the unencrypted B2I and B3I signals have earlier been revealed from analysis of the M1 signals (see Gao et al. 2009 and references therein). Building up on these analyses, various manufacturers of geodetic multi-GNSS receivers have identified the proper code generator configuration to replicate the pseudo-random number codes transmitted by all other satellites in the constellation. This work has previously enabled researchers to track BeiDou signals in all frequency bands, to compute precise orbit and clock products (Shi et al 2012, Steigenberger 2013, He et al. 2013), to study the signal performance and to conduct initial positioning experiments (Shi et al 2013, Montenbruck et al. 2013).

With the recent availability of a public ICD, orbit and clock information as well as auxiliary data are now available to real-time users thus enabling the computation of instantaneous positioning solutions. Within this paper we first provide a summary of the BeiDou navigation message contents and describe key similarities and differences with respect to that of GPS. Special attention is given to group delay parameters that are required for dual-frequency users but only partly covered by the present B1I signal ICD. Thereafter the performance of the navigation message is assessed based on external comparisons and the achievable positioning accuracy is highlighted based on practical tests with actual data.

Observation data and navigation messages for use within this study have been recorded since mid January 2013 with various Trimble NetR9, Septentrio AsteRx3 and Triumph DeltaG3TH receivers of the Cooperative Network for GNSS Observation (CONGO) and the MultiGNSS Experiment (MGEX) network of the International GNSS Service (IGS) (Montenbruck 2012, Rizos et al. 2013). 


\section{BEIDOU MESSAGE TYPES AND STRUCTURE}

The BeiDou system utilizes two different forms of navigation messages (named as D1 and D2), which are transmitted by the MEO/IGSO and GEO satellites, respectively (CSNO 2012). Similar to GPS, all messages are based on 30-bit words and subframes with a length of 10 words (300 bits). However, a different parity concept enabling single-bit error correction per 15-bit half-word is employed. Along with this, the bits of two half-words in a given word are interleaved and must thus be de-interleaved upon reception for proper interpretation of the data. As a sidenote, we mention that both interleaved (i.e. unmodified) and de-interleaved versions of the raw navigation data frames are output by current receivers depending on their brand and firmware version. Care must thus be taken in the decoding of such data as well as their possible use for data bit removal in radio science applications (Beyerle et al. 2009) and assisted weak signal tracking.

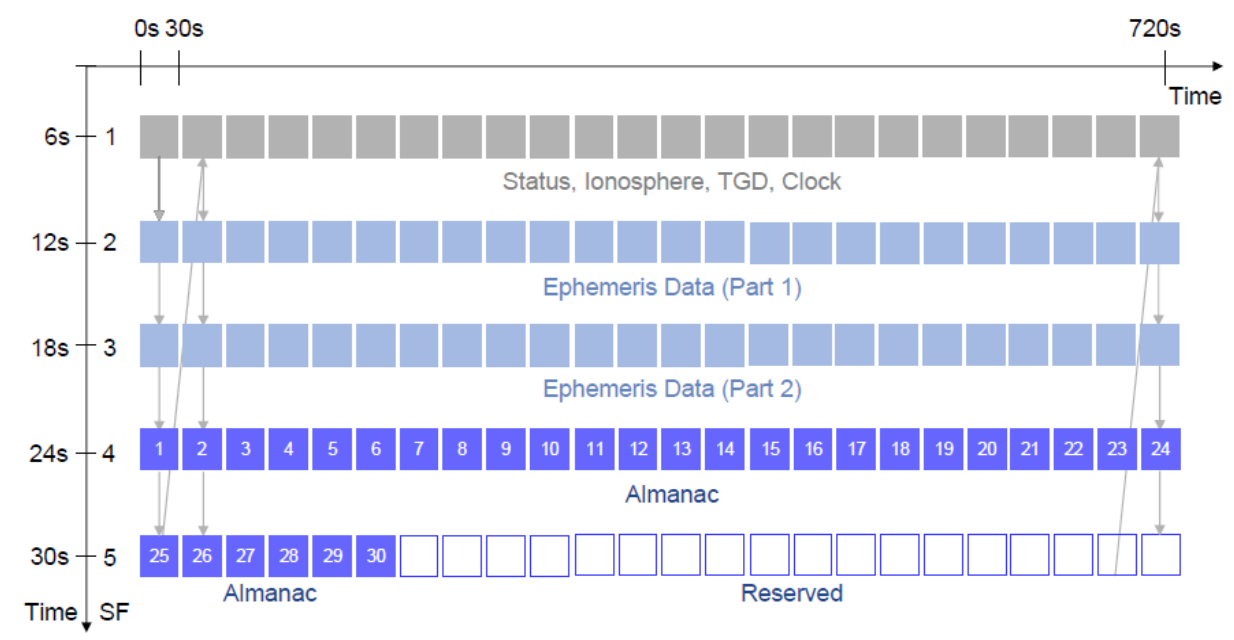

Figure 1. Frame structure of the BeiDou D1 navigation message for MEO/IGSO satellites. Each block represents a single subframe. Numbers for almanac data indicate the space vehicle ID.

The contents and structure of the D1 navigation messages closely resembles that of the GPS message and is transmitted with the same data rate of $50 \mathrm{bits} / \mathrm{s}$. Basic ephemeris data of the transmitting satellite are comprised in the first three subframes of each frame and repeated once every $30 \mathrm{~s}$ (Figure 1). The contents of the 4th and 5th subframe is subcommutated 24 times before the entire message contents is repeated. The respective pages provide almanac information for up to 30 satellites as well as ancillary data such as UTC and inter-system time offsets that are not immediately required for the computation of a position fix. 18 pages of frame 15 are presently reserved for future use. A full superframe (comprising 24 frames of 5 subframes, each) is transmitted in 12 minutes (i.e. 0.5 min less than in case of GPS).

The D2 navigation message broadcast by the geostationary BeiDou satellites employs a ten times higher rate of $500 \mathrm{bits} / \mathrm{s}$ and a single subframe is thus transmitted in $0.6 \mathrm{~s}$ (Figure 2). However, basic navigation data (such as ephemeris and clock information) are split across a sequence of ten pages of subframe 1, which results in the same repeat rate of $30 \mathrm{~s}$ as for the MEO/IGSO satellites. Only 150 out of the available 300 bits in each subframe 1 are presently used for transmission of the basic GEO navigation data.

Subframes 2 and 3 provide pseudorange corrections of up to 18 satellites for regional users along with related accuracy information. The information in each subframe is distributed 
across 6 pages and repeated once every $18 \mathrm{~s}$. For subframe 4 a similar structure is foreseen, but no data are presently defined in the ICD. Subframe 5, finally, contains the BeiDou almanac, ionosphere grid data for regional users and intersystem time offsets. The respective information is split across a total of 120 different pages and repeated once every $6 \mathrm{~min}$. The corrections and ionosphere data are intended for users within a longitude range of $70^{\circ}-145^{\circ}$ and a latitude range of about $5^{\circ}-55^{\circ}$.

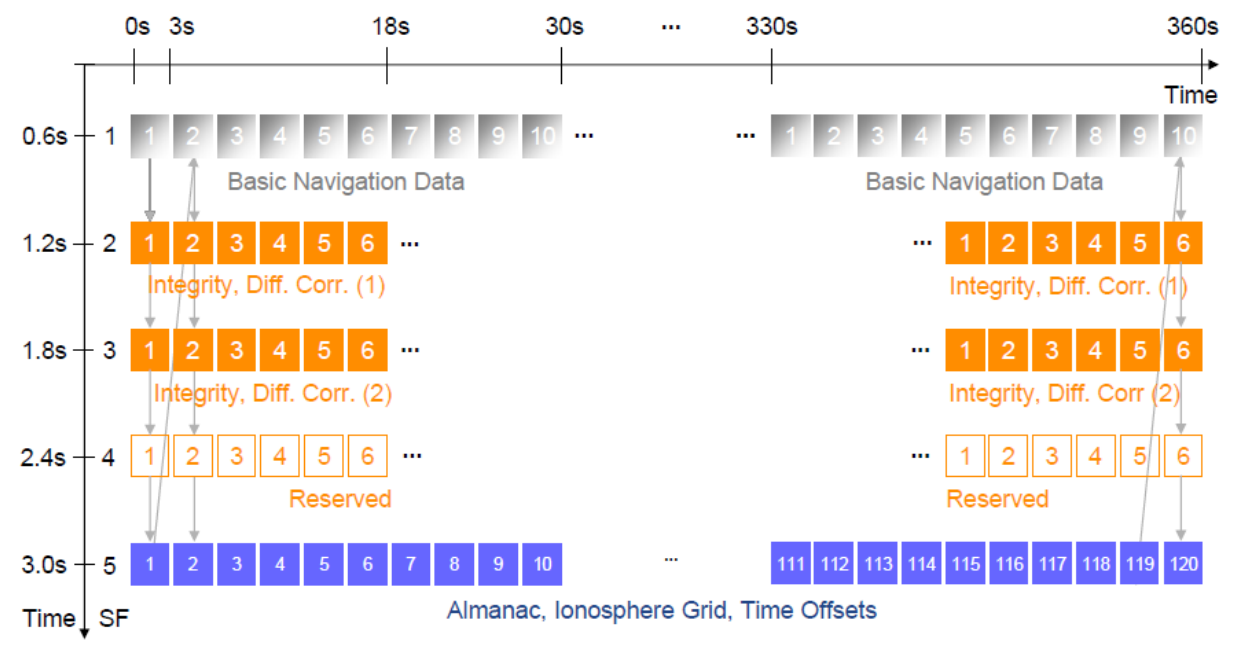

Figure 2. Frame and page structure of the D2 navigation message for BeiDou GEO satellites. Each block represents a single subframe. Numbers indicate the page number of the individual data types.

The GEO satellites can thus be considered as a satellite based augmentation system (SBAS) offering increased navigation accuracy for single-frequency BeiDou users in this region. The availability of augmentation data appears of special interest for mass market users throughout China and its neighbourhood, even though the quality of the SBAS-data and its benefit for single-frequency positioning could not be assessed within the present study.

Table 2 Comparison of bit numbers and scaling of broadcast orbit and clock parameters applied in the BeiDou, GPS (IS-GPS-200 2011) and Galileo systems (EU 2010). Void entries indicate consistency with GPS.

\begin{tabular}{lcccccc}
\hline Parameter & \multicolumn{2}{c}{ BeiDou } & \multicolumn{2}{c}{ GPS } & \multicolumn{2}{c}{ Galileo } \\
& Bits & Scale & Bits & Scale & Bits & Scale \\
\hline Clock offset $\left(\mathrm{a}_{0}\right)$ & 24 & $2^{-33}$ & 22 & $2^{-31}$ & 31 & $2^{-34}$ \\
Clock drift $\left(\mathrm{a}_{1}\right)$ & 22 & $2^{-50}$ & 16 & $2^{-43}$ & 21 & $2^{-46}$ \\
Clock acceleration $\left(\mathrm{a}_{2}\right)$ & 11 & $2^{-66}$ & 8 & $2^{-55}$ & 6 & $2^{-59}$ \\
Semi-major axis $(\sqrt{\mathrm{a}})$ & & & 32 & $2^{-19}$ & & \\
Eccentricity $(\mathrm{e})$ & & & 32 & $2^{-33}$ & & \\
Inclination $(\mathrm{i})$ & & & 32 & $2^{-31}$ & & \\
Argument of perigee $(\omega)$ & & & 32 & $2^{-31}$ & & \\
Ascending node $(\Omega)$ & & & 32 & $2^{-31}$ & & \\
Mean anomaly $(\mathrm{M})$ & & & 32 & $2^{-31}$ & & \\
Mean motion difference $(\Delta \mathrm{n})$ & & & 16 & $2^{-43}$ & & \\
Inclination rate $(\mathrm{di} / \mathrm{dt})$ & & & 14 & $2^{-43}$ & & \\
Nodal rate $(\mathrm{d} \Omega / \mathrm{dt})$ & 18 & $2^{-31}$ & 16 & $2^{-43}$ & & \\
Periodic angle corrections $\left(\mathrm{C}_{\mathrm{u}}, \mathrm{C}_{\mathrm{i}}\right)$ & 18 & $2^{-29}$ & & \\
Periodic range corrections $\left(\mathrm{C}_{\mathrm{r}}\right)$ & 18 & $2^{-6}$ & 16 & $2^{-5}$ & & \\
\hline
\end{tabular}


The specific choice of data bits and scaling factors for the BeiDou ephemeris parameters in the D1 and D2 navigation messages is compared in Table 2with those of GPS and Galileo. Except for the harmonic perturbations in radius, argument of latitude and inclination that cover a four times higher range of values but equal resolution, the representation of all orbit related parameters matches that of GPS (and Galileo). A larger number of data bits is foreseen for the clock offset polynomial which results in a higher range of values for the constant clock offset term and a better resolution for the first and second order derivatives. This choice is obviously justified by a high stability of the BeiDou Rubidium clock that has been reported in recent investigatons (Hauschild et al. 2012b).

\section{BEIDOU BROADCAST NAVIGATION MODELS}

\subsection{Orbit Model}

BeiDou is the first navigation system employing MEO, IGSO, and GEO satellites in a common constellation. While the analytical GPS model is well accepted for medium altitude orbits (and, in case of QZSS also inclined geosynchronous orbits), the numerical integration of an epoch state vector has been favored for traditional SBAS satellites in geostationary orbit. However, GEO satellites do not primarily serve to communicate correction data in BeiDou, but are an integral part of the navigation system architecture. As such, a common set of orbital parameters has been preferred for all classes of satellites. However, a slightly varied orbital model has been defined for geostationary BeiDou satellites to cope with the (near-) singularity of the Keplerian elements representation for low inclinations. The difference affects the final transformation of the position from the orbital plane to Earth-fixed coordinates. This is usually described as a rotation about the $x$-axis by the inclination angle $i$ and a subsequent rotation about the $z$-axis by the instantaneous longitude of the ascending node $\lambda_{\Omega}$ :

$$
\left(\begin{array}{l}
x \\
y \\
z
\end{array}\right)=\boldsymbol{R}_{z}\left(-\lambda_{\Omega}\right) \cdot \boldsymbol{R}_{x}(-i) \cdot\left(\begin{array}{c}
r \cos (u) \\
r \sin (u) \\
0
\end{array}\right) .
$$

Given the longitude $\Omega_{0}$ of the ascending node at the ephemeris reference epoch $t_{0 e}$ (but expressed relative to the Earth orientation at the beginning of the week $t_{0 w}$ ) as provided in the broadcast ephemeris, the longitude of the ascending node at the epoch of interest $t$ is obtained by accounting for the inertial rotation of the orbital plane and the Earth rotation:

$$
\lambda_{\Omega}(t)=\left(\Omega_{0}+\dot{\Omega} \cdot\left(t-t_{0 e}\right)\right)-\omega_{\oplus} \cdot\left(t-t_{0 w}\right) .
$$

For use with geostationary BeiDou satellites, the model is modified by referring the inclination to an auxiliary plane with a $5^{\circ}$ tilt relative to the Earth equator and by adopting a different reference direction for measuring the longitude of the ascending node. This results in the modified transformation

$$
\left(\begin{array}{c}
x \\
y \\
z
\end{array}\right)=\boldsymbol{R}_{z}\left(\omega_{\oplus} \cdot\left(t-t_{0 w}\right)\right) \cdot \boldsymbol{R}_{x}\left(-5^{\circ}\right) \cdot \boldsymbol{R}_{z}(-\Omega) \cdot \boldsymbol{R}_{x}(-i) \cdot\left(\begin{array}{c}
r \cos (u) \\
r \sin (u) \\
0
\end{array}\right)
$$


with

$$
\Omega(t)=\left(\Omega_{0}+\dot{\Omega} \cdot\left(t-t_{0 e}\right)\right)-\omega_{\oplus} \cdot\left(t_{0 e}-t_{0 w}\right)
$$

By introducing the auxiliary plane, singularities of the legacy model can effectively be avoided. In practice, the orbital inclination of the geostationary BeiDou satellites relative to the Earth equator is confined to less than about $2^{\circ}$, so that the inclination relative to the auxiliary plane is sufficiently different from zero at all times.

For completeness, it is emphasized that BeiDou employs different values for the Earth gravitational coefficient and the Earth rotation rate. A summary of the respective values in BeiDou, GPS, and Galileo is provided in Table 3. Use of the constellation-specific values is mandatory to ensure full accuracy of the broadcast ephemerides in real-time positioning applications.

Table 3 Physical parameters of BeiDou, GPS, and Galileo ephemeris models.

\begin{tabular}{lccc}
\hline Parameter & BeiDou & GPS & Galileo \\
\hline $\mathrm{GM}_{\oplus}$ & $398600.4418 \cdot 10^{9} \mathrm{~m}^{3} / \mathrm{s}^{2}$ & $398600.5 \cdot 10^{9} \mathrm{~m}^{3} / \mathrm{s}^{2}$ & $398600.4418 \cdot 10^{9} \mathrm{~m}^{3} / \mathrm{s}^{2}$ \\
$\omega_{\oplus}$ & $7.2921150 \cdot 10^{-5} \mathrm{rad} / \mathrm{s}$ & $7.2921151467 \cdot 10^{-5} \mathrm{rad} / \mathrm{s}$ & $7.2921151467 \cdot 10^{-5} \mathrm{rad} / \mathrm{s}$ \\
\hline
\end{tabular}

\subsection{Clock Offsets and Timing Group Delays}

The clock model employed in BeiDou is essentially the same as that of GPS and Galileo and describes the satellite clock offset as the sum of a second-order polynomial in time and a periodic relativistic correction depending on the eccentric anomaly.

Care must be taken, however, that the clock reference is chosen in a different manner than for other GNSS constellations. Based on the example of GPS, it has become common practice to define clock offsets in both broadcast and precise ephemeris products with respect a ionosphere-free dual-frequency combination of conventional reference signals (such as L1/L2 P(Y)-code for GPS; see Montenbruck and Hauschild 2013), which is itself considered to be free of group delays. In contrast to this, the BeiDou clock offsets provided in the broadcast navigation message are referred to a single-frequency B3 signal (Wu et al. 2013). As a consequence, differential code biases (DCBs) need to be applied in both in B1 and B2 singlefrequency navigation as well as B1/B2 or B1/B3 dual-frequency navigation.

The respective observation model for the pseudoranges $P$ measured on the individual frequencies is given by

$$
\begin{array}{ll}
P_{\mathrm{B} 1}=\rho+c \delta t^{\mathrm{rcv}}-c \delta t^{\mathrm{sat}}+T+I_{\mathrm{B} 1} & +\mathrm{DCB}_{\mathrm{B} 1-\mathrm{B} 3} \\
P_{\mathrm{B} 2}=\rho+c \delta t^{\mathrm{rcv}}-c \delta t^{\mathrm{sat}}+T+I_{\mathrm{B} 1} \cdot\left(f_{\mathrm{B} 1}^{2} / f_{\mathrm{B} 2}^{2}\right) & +\mathrm{DCB}_{\mathrm{B} 2-\mathrm{B} 3}, \\
P_{\mathrm{B} 3}=\rho+c \delta t^{\mathrm{rcv}}-c \delta t^{\mathrm{sat}}+T+I_{\mathrm{B} 1} \cdot\left(f_{\mathrm{B} 1}^{2} / f_{\mathrm{B} 3}^{2}\right) &
\end{array}
$$

where $\rho$ denotes the geometric range, $\delta t^{\text {sat }}$ and $\delta t^{\text {rcv }}$ are the satellite and receiver clock offsets and $T$ and $I$ describe the tropospheric and ionospheric path delays. Accordingly, a bias 


$$
\mathrm{DCB}_{\mathrm{IF}(\mathrm{B} 1, \mathrm{~B} 2)}=\frac{f_{\mathrm{B} 1}^{2}}{f_{\mathrm{B} 1}^{2}-f_{\mathrm{B} 2}^{2}} \cdot \mathrm{DCB}_{\mathrm{B} 1-\mathrm{B} 3}-\frac{f_{\mathrm{B} 2}^{2}}{f_{\mathrm{B} 1}^{2}-f_{\mathrm{B} 2}^{2}} \cdot \mathrm{DCB}_{\mathrm{B} 2-\mathrm{B} 3}
$$

needs to be considered, when processing a ionosphere-free combination of B1 and B2 pseudoranges. Dual-frequency B1/B3 users, in contrast, just need to apply a scaled version of the B1-B3 code bias.

$$
\mathrm{DCB}_{\mathrm{IF}(\mathrm{B} 1, \mathrm{~B} 3)}=\frac{f_{\mathrm{B} 1}^{2}}{f_{\mathrm{B} 1}^{2}-f_{\mathrm{B} 3}^{2}} \cdot \mathrm{DCB}_{\mathrm{B} 1-\mathrm{B} 3}
$$

The B1I Open Service ICD presently defines a single "equipment group delay differential" $\mathrm{T}_{\mathrm{GD} 1}$ that shall be applied by single-frequency $\mathrm{B} 1$ users and needs to be subtracted from the broadcast clock offset. From comparison with Equation (5), the $\mathrm{T}_{\mathrm{GD} 1}$ parameter is found to match the B1-B3 differential code bias introduced above. Despite a similarity of names and a similar application in single-frequency navigation, users should be well aware, though, that the BeiDou $\mathrm{T}_{\mathrm{GD} 1}$ group delay parameter is conceptually different from the common "Timing Group Delay" parameter T $\mathrm{GD}_{\mathrm{G}}$ employed in GPS (cf. Montenbruck and Hauschild 2013). This difference is vital for a proper processing of other observations than B1I as well as linear combinations of code observations on multiple frequencies.

Evidently, a second group delay parameter is required when working with B2 singlefrequency or B1/B2 dual-frequency observations. In fact, a placeholder for a $\mathrm{T}_{\mathrm{GD} 2}(\mathrm{~B} 2-\mathrm{B} 3)$ parameter has already been proposed by Chinese authorities for the BeiDou extension of the Receiver INdependent EXchange format (RINEX; IGS RWG 2013), but no such parameter is presently specified in the B1 Open Service ICD. On the other hand, inspection of the raw BeiDou navigation data frames shows that a reserved 10-bit data field next to the $T_{G D 1}$ parameter in subframe 1 of the D1 message (and likewise page 1 of suframe 1 in the D2 message) is in fact populated with data that might be interpreted as the missing $\mathrm{T}_{\mathrm{GD} 2}$ parameter.
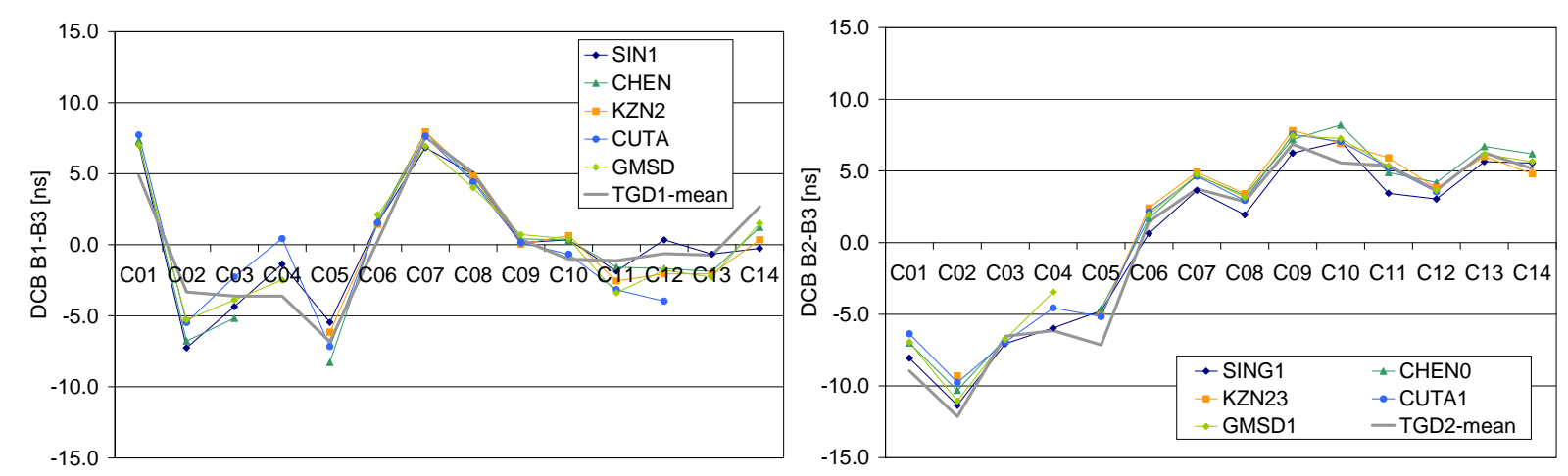

Figure 3. Comparison of broadcast $\mathrm{T}_{\mathrm{GD} 1}$ and $\mathrm{T}_{\mathrm{GD} 2}$ group delay parameters with $\mathrm{B} 1-\mathrm{B} 3$ and $\mathrm{B} 2-\mathrm{B} 3$ differential code biases deduced from triple-frequency observations of CONGO and MGEX monitoring stations in $13 \mathrm{Feb}$. 2013. For comparison all values have been normalized to a zero constellation average.

To validate this suspicion, we compared the extracted $\mathrm{T}_{\mathrm{GD} 2}$ values with differential code biases derived from triple-frequency observations of monitoring stations in the Asia-Pacific area. Daily averages of inter-frequency pseudorange differences were formed to obtain the B1I-B3I, B2I-B3I, and B1I-B2I differential code biases for each station after compensating 
the frequency-dependent ionospheric path delays through global ionosphere maps (GIMs) provided by the IGS)

As shown in Figure 3, the observations clearly support the interpretation of $\mathrm{T}_{\mathrm{GD} 1}$ as a B1-B3 differential code bias. More importantly, they provide clear evidence that a $\mathrm{T}_{\mathrm{GD} 2}$ parameter matching the B2-B3 differential code bias is indeed provided in the undocumented bit fields next to $\mathrm{T}_{\mathrm{GD} 1}$. This enables all dual-frequency users to properly account for intrinsic group delays when using a ionosphere-free B1/B2 combination for real-time positioning.

\subsection{Ionosphere Model}

For single-frequency users, BeiDou broadcasts a set of eight correction parameters for the computation of ionospheric path delays. The underlying model resembles the well established GPS Klobuchar model but is formulated in terms of geographic (rather than geomagnetic) coordinates and uses a rigorous thin-layer mapping function (CSNO 2012, Wu et al. 2013). Variations of the vertical range delay on the day side are described by a latitude-dependent, periodic function in local time, while a constant delay of $5 \mathrm{~ns}$ is applied on the night side. Coefficients for the BeiDou ionosphere model are determined from monitoring stations in the China mainland ( $\mathrm{Wu}$ et al 2013) and updated once every two hours. Following Wu et al. (2013) the BeiDou model outperforms the Klobuchar model for northern hemisphere users in the Asia-Pacific region but exhibits a degraded performance outside this area.

\section{EPHEMERIS PERFORMANCE ASSESSMENT}

\subsection{Ephemeris Age}

The BeiDou navigation message comprises an integer-valued issue-of-data-ephemeris parameter (IODE) which signifies the age of the satellite's orbit information in hours. For geostationary satellites, which are in permanent contact with the control station, the always exhibit the minimum age of one hour. However, the age-of-data for the non-geostationary satellites varies with the satellite location, since uplink stations of the BeiDou ground segment are limited to the China mainland.

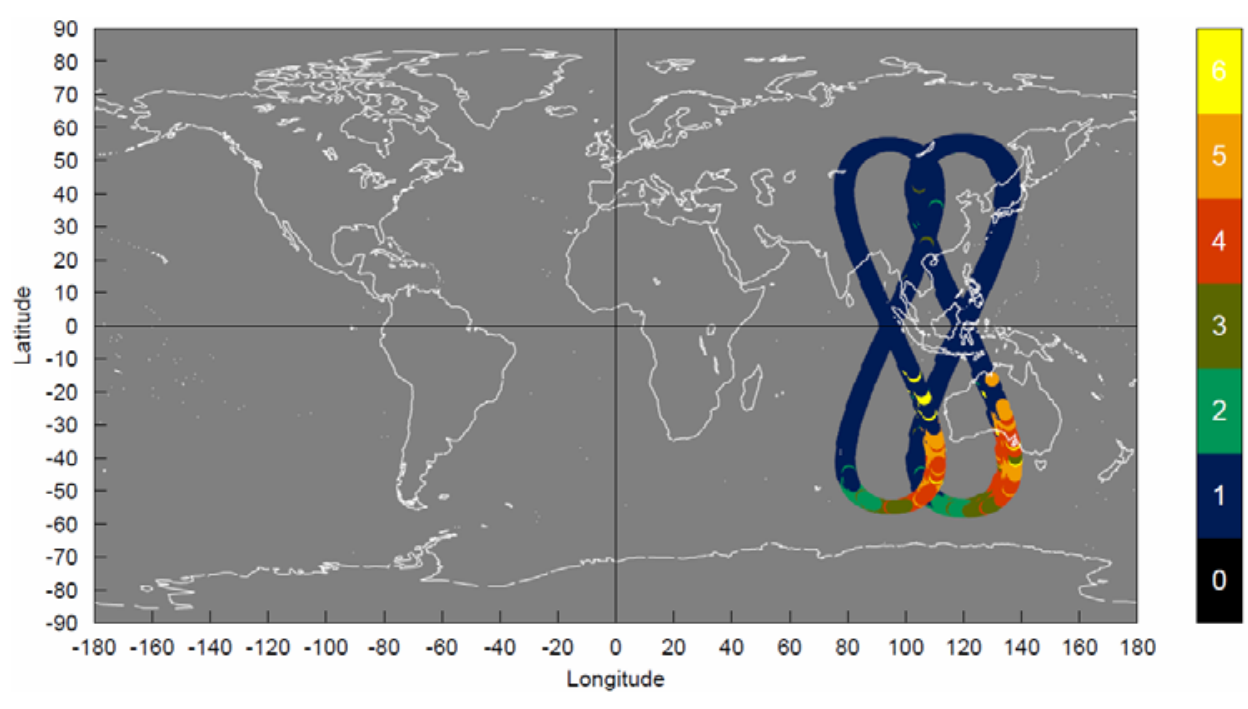

Figure 4. Age of received ephemeris information as a function of the satellite's ground-position for BeiDou IGSO satellites 
This is illustrated in Figures 4-5, which show the IODE values of newly received navigation messages as a function of the satellite's foot print at the time of reception. In case of IGSO satellites, the age-of-data is mostly within the 1-hour range except for the south-eastern portion of the figure-for-eight ground-track (Figure 4). Here, the ephemeris data may exhibit an age of up 6 hours, implying a possible degradation of the user navigation accuracy in Australia.

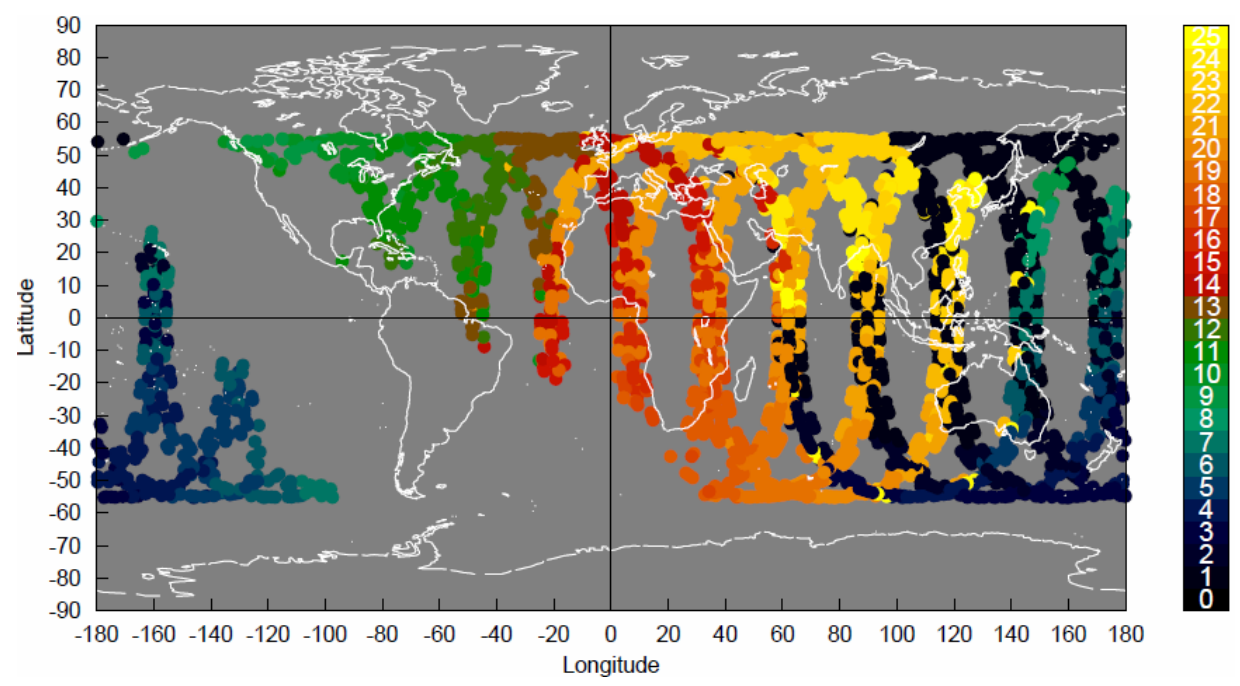

Figure 5. Age of received ephemeris information as a function of the satellite's ground-position for BeiDou MEO satellites. Due to a lack of world-wide monitoring stations available for this study, no data could be collected in a belt ranging from the north-Pacific to the south-Atlantic.

For MEO satellites, this effect is even more pronounced and latencies of up to $24 \mathrm{~h}$ are routinely observed. As can be recognized from Figure 5, the age-of-ephemeris increases in an eastern direction away from China and is generally highest before the satellite approaches China from the West. New ephemeris information becomes available when the ground track passes between $60^{\circ} \mathrm{E}$ and $130^{\circ} \mathrm{E}$. In addition, it may be recognized that MEO satellites on a descending ground track in this region benefit from earlier updates than satellites on an ascending ground track.

\subsection{Satellite Laser Ranging Validation}

As a first test of the BeiDou broadcast ephemeris accuracy, satellite laser ranging measurements collected by the International Laser Ranging Service (ILRS, Pearlman et al 2002) have been compared with orbits computed from the navigation messages. SLR reflector offsets with respect to the satellite's center-of-mass (CoM) have been accounted based on values distributed by the ILRS and assuming a nominal, yaw-steering attitude law. Even though the broadcast orbits are expected to refer to an adopted GNSS antenna phase center, the respective offset from the CoM is unknown and could not be considered in the SLR analysis. The associated modelling errors will primarily show up in a mean bias of the same order as the radial antenna offset.

Overall, the SLR residuals of the currently tracked satellites exhibit a standard deviation of 0.5-0.7 $\mathrm{m}$ (Figure 6), which provides a direct measure for the contribution of orbit errors to the overall signal-in-space range error (SISRE) of the BeiDou broadcast ephemerides. Interestingly, the observed bias of the SLR residuals is much smaller than would be expected for an ephemeris that is referenced to the antenna phase center rather than the center-of-mass. 

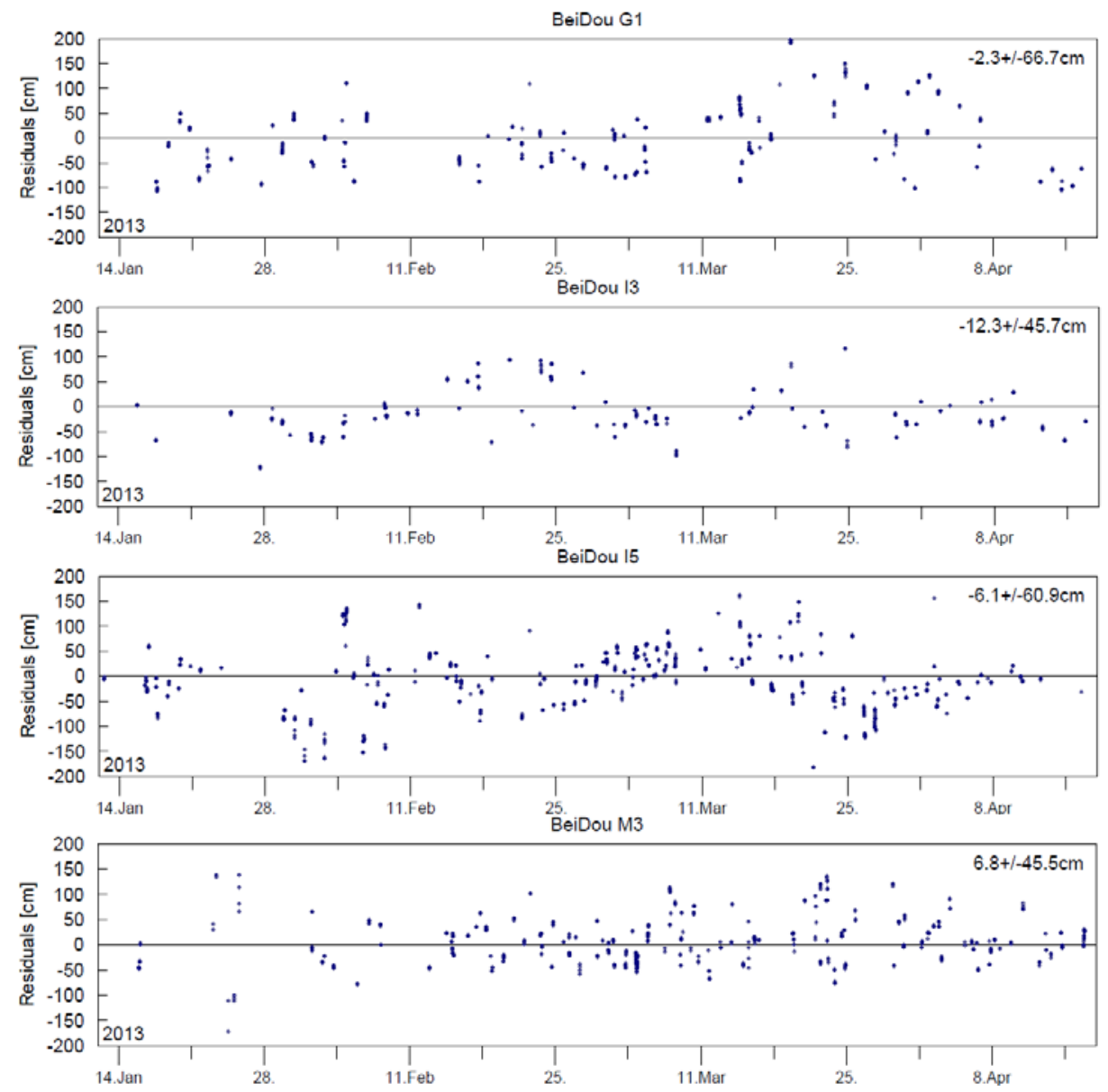

Figure 6. Satellite laser ranging residuals (observed-values minus-modelled range based on broadcast orbits) for BeiDou satellites tracked by the ILRS.

\subsection{Comparison with Postprocessed Orbit and Clock Products}

For a complementary quality assessment, BeiDou orbit and clock products have been computed from a small network of monitoring stations operated as part of the CONGO and MGEX networks. For the regional (IGSO, GEO) component five to six stations in the AsiaPacific region were employed (Montenbruck et al. 2012). The MEO satellites are also tracked by various stations in Europe, Africa and America (Rizos et al. 2013), but large visibility gaps remain and a fully global coverage is not yet achievable with the current networks. The orbit and clock determination makes use of a ionosphere-free linear combination of BeiDou observations in the B1/B2 band and builds-up on a priori estimates of station coordinates, receiver clock offsets and tropospheric delays derived from a GPS-only positioning. Details of the employed processing concept are described in (Steigenberger et al. 2013).

Orbit differences of MEO and IGSO satellites in radial, along-track and cross-track direction are shown in Figure 7 for 10-day period in spring 2013. GEO satellites have intentionally been excluded from the comparison since the quasi-static viewing geometry and the sparse network do not presently enable a highly accurate orbit determination for this part of the constellation. For the post-processed orbits of non-GEO satellites, 3D rms position accuracies of about $0.5 \mathrm{~m}$ (MEOs) and $0.9 \mathrm{~m}$ (IGSOs) have been assessed based on day-boundary discontinuities while overlap tests indicate an internal consistency of consecutive 3-day orbit solutions at the 0.1-0.2 m level. 

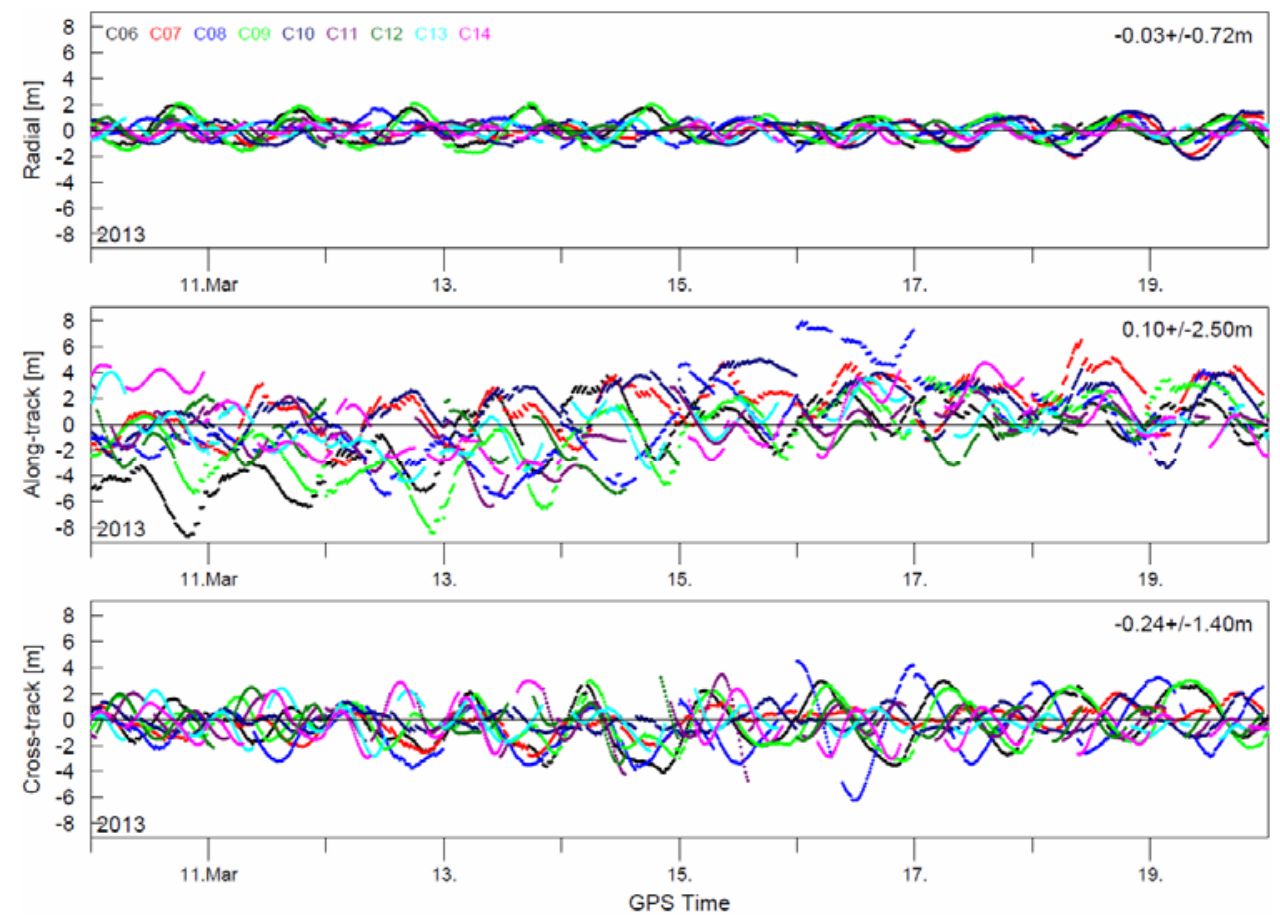

Figure 7. Differences between BeiDou MEO and IGSO broadcast orbits and post-processed products

The comparison indicates an overall accuracy of better than $3 \mathrm{~m}$ (3D rms) for the MEO/IGSO broadcast ephemerides and a radial position error of well below $1 \mathrm{~m}$. Detailed statistics of the difference between broadcast ephemerides and post-processed orbits for the test period are summarized in Table 4. Obviously, no systematic bias exists between the radial positions of the two types of orbit products, which again suggests that the broadcast ephemerides refer to a position close to the spacecraft center-of-mass.

Table 4 Differences (mean \pm standard deviation) between broadcast ephemerides and post-processed products for BeiDou MEO and IGSO satellites (March 10-19, 2013).

\begin{tabular}{lccc}
\hline Component & MEO & IGSO & MEO+IGSO \\
\hline Radial & $-0.02 \pm 0.44 \mathrm{~m}$ & $-0.04 \pm 0.83 \mathrm{~m}$ & $-0.03 \pm 0.72 \mathrm{~m}$ \\
Along-track & $-0.28 \pm 1.96 \mathrm{~m}$ & $+0.29-2.72 \mathrm{~m}$ & $+0.10 \pm 2.50 \mathrm{~m}$ \\
Cross-track & $-0.20 \pm 1.22 \mathrm{~m}$ & $-0.27 \pm 1.49 \mathrm{~m}$ & $-0.24 \pm 1.40 \mathrm{~m}$ \\
Clock & & & $16.95 \pm 1.45 \mathrm{~m}$ \\
Radial-(clock-ensemble) & & & $-0.03 \pm 1.20 \mathrm{~m}$ \\
\hline
\end{tabular}

For a comparison of broadcast clocks with post-processed solutions, care must be taken that the respective values are referred to different signals as well as to different realization of the BeiDou system time. As discussed in Section 3.2, the broadcast clocks are referenced to single-frequency B3 observations, while the post-processed clock products provided for the present analysis are based on ionosphere-free B1/B2 observations. To account for satellitedependent inter-frequency and inter-signal biases, the satellite contribution to the differential code bias $\mathrm{DCB}_{\mathrm{IF}(\mathrm{B} 1, \mathrm{~B} 2)}$ (cf. Equation (6)) has been compensated based on the $\mathrm{T}_{\mathrm{GD} 1}$ and $\mathrm{T}_{\mathrm{GD} 2}$ group delay parameters provided in the navigation message. While the uncorrected broadcastminus-post-processed clock difference exhibits a standard deviation of about $3 \mathrm{~m}$, the DCB correction results in a two-times better consistency. A remaining bias of about $17 \mathrm{~m}$ (see Table 4) reflects different GPS-BDS inter-system biases in the ground receiver network used 
to generate the respective orbit products. As a result of this bias a corresponding difference of roughly $60 \mathrm{~ns}$ can be expected for the GPS-BDS time offset as determined by the different processing systems.

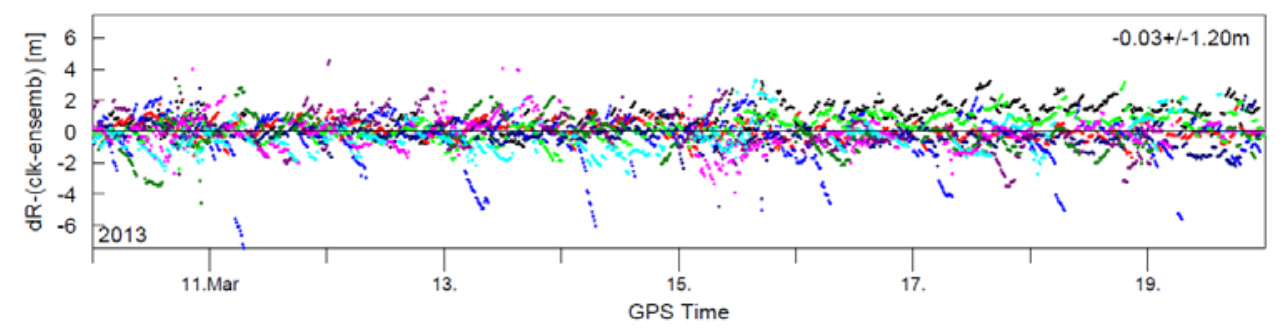

Figure 8. Combined contribution of radial orbit errors and clock errors to the user range error in the comparison of BeiDou MEO and IGSO broadcast orbits with post-processed products

In view of the GNSS measurement principle, radial orbit errors and satellite clock errors exhibit a notable correlation and only the difference of both quantities contributes to the overall signal-in-space range error (SISRE). In comparison with our post-processed ephemeris products a standard deviation of about $1.20 \mathrm{~m}$ is obtained for the combined contribution of radial orbit and clock errors, which is slightly lower than for the clock error alone. It must be noted, though, that the post-processed clock solution suffers from numerous discontinuities as a result of interrupted visibility and tracking in the sparse monitoring network.

Combining clock and radial orbit errors $(\Delta(R-c d t))$ with a weighted sum of normal and cross-track errors $(\Delta T, \Delta N)$, an overall SISRE of

$$
\mathrm{SISRE}=\sqrt{\sigma_{\Delta(R-c d t)}^{2}+\gamma^{2}\left(\sigma_{\Delta T}^{2}+\sigma_{\Delta N}^{2}\right)}
$$

is obtained. Here $\gamma=\overline{\sin (\varphi)} \approx \bar{\varphi}$ denotes the mean angle between the line-of-sight and the radial direction for a terrestrial observer. A value of $\gamma=7$ is commonly adopted for SISRE analysis of MEO GNSS satellites (Warren and Raquet 2003), while $\gamma=11$ is appropriate for geosynchronous (IGSO and GEO) satellites in view of their larger orbital radius. Based on the results of Table 4, a SISRE of 1.2-1.3 m can be inferred from the comparison of broadcast and post-processed ephemeris products. It must be emphasized, though, that this value represents a conservative upper bound for the quality of BeiDou broadcast ephemerides due to the limited accuracy of the employed reference products. For comparison, an average SISRE of $0.8 \mathrm{~m}$ is currently reported for the entire GPS constellation (Gruber 2012), while values of $0.6 \mathrm{~m}$ and $0.3 \mathrm{~m}$ are obtained when considering only Block IIR and IIF satellites.

\subsection{Positioning Performance}

We conclude this section with a comparison of pseudorange-based single point positioning results using GPS and BeiDou observations. Results for a total of five multi-GNSS monitoring stations in the Asia-Pacific region are presented in Table 5. Raw observations on up to three frequencies as well as broadcast ephemerides have been collected over a $24 \mathrm{~h}$ data arc. Single-point position fixes based on both single-frequency pseudoranges as well as ionosphere-free dual-frequency combinations were then computed in post-processing. For compensation of ionospheric path delays, global ionosphere maps (GIMs) provided by the IGS were employed. Differential code biases were applied as required for the individual 
signals or signal combinations. In case of BeiDou, broadcast group delay parameters were adopted while GPS-related DCBs were again provided by the IGS.

Table 5. RMS errors and PDOP of code-based GPS-only and BeiDou-only position fixes based on broadcast ephemerides for 15 Feb 2013 ( $E=E a s t, N=N o r t h, U=U p, 3 D=3 D$ position error). Ionospheric path delays in single-frequency navigation solutions were corrected using global ionosphere maps. Observations were processed down to a minimum elevation of $5^{\circ}$.

\begin{tabular}{lccccccccc}
\hline Station & & GPS & GPS & BDS & BDS & BDS & BDS & BDS & PDOP \\
(ID, Receiver) & & L1 & L1/L2 & B1 & B2 & B3 & B1/B2 & B1/B3 & BDS \\
\hline Singapore & E & $0.70 \mathrm{~m}$ & $0.85 \mathrm{~m}$ & $0.78 \mathrm{~m}$ & $0.89 \mathrm{~m}$ & $0.90 \mathrm{~m}$ & $1.36 \mathrm{~m}$ & $1.14 \mathrm{~m}$ & \\
(SIN1,NetR9) & $\mathrm{N}$ & $0.87 \mathrm{~m}$ & $0.59 \mathrm{~m}$ & $0.98 \mathrm{~m}$ & $1.35 \mathrm{~m}$ & $1.22 \mathrm{~m}$ & $0.93 \mathrm{~m}$ & $1.13 \mathrm{~m}$ & \\
& $\mathrm{U}$ & $1.71 \mathrm{~m}$ & $2.04 \mathrm{~m}$ & $2.16 \mathrm{~m}$ & $3.50 \mathrm{~m}$ & $3.52 \mathrm{~m}$ & $2.34 \mathrm{~m}$ & $3.13 \mathrm{~m}$ & \\
& 3D & $2.04 \mathrm{~m}$ & $2.29 \mathrm{~m}$ & $2.50 \mathrm{~m}$ & $3.86 \mathrm{~m}$ & $3.83 \mathrm{~m}$ & $2.86 \mathrm{~m}$ & $3.52 \mathrm{~m}$ & 1.9 \\
Tanegashima & $\mathrm{E}$ & $0.86 \mathrm{~m}$ & $0.80 \mathrm{~m}$ & $0.90 \mathrm{~m}$ & $1.03 \mathrm{~m}$ & $1.07 \mathrm{~m}$ & $1.43 \mathrm{~m}$ & $1.66 \mathrm{~m}$ & \\
(GMSD,NetR9) & $\mathrm{N}$ & $1.24 \mathrm{~m}$ & $0.99 \mathrm{~m}$ & $1.28 \mathrm{~m}$ & $1.78 \mathrm{~m}$ & $1.59 \mathrm{~m}$ & $1.57 \mathrm{~m}$ & $1.89 \mathrm{~m}$ & \\
& $\mathrm{U}$ & $3.09 \mathrm{~m}$ & $2.01 \mathrm{~m}$ & $3.06 \mathrm{~m}$ & $3.98 \mathrm{~m}$ & $3.90 \mathrm{~m}$ & $4.32 \mathrm{~m}$ & $3.95 \mathrm{~m}$ & \\
& 3D & $3.44 \mathrm{~m}$ & $2.38 \mathrm{~m}$ & $3.44 \mathrm{~m}$ & $4.48 \mathrm{~m}$ & $4.34 \mathrm{~m}$ & $4.81 \mathrm{~m}$ & $4.68 \mathrm{~m}$ & 2.6 \\
Sydney & $\mathrm{E}$ & $0.79 \mathrm{~m}$ & $0.64 \mathrm{~m}$ & $2.51 \mathrm{~m}$ & $2.39 \mathrm{~m}$ & $\mathrm{n} / \mathrm{a}$ & $3.36 \mathrm{~m}$ & $\mathrm{n} / \mathrm{a}$ & \\
(UNX3,AsteRx3) & $\mathrm{N}$ & $0.76 \mathrm{~m}$ & $0.73 \mathrm{~m}$ & $1.28 \mathrm{~m}$ & $1.26 \mathrm{~m}$ & & $2.24 \mathrm{~m}$ & & \\
& $\mathrm{U}$ & $1.50 \mathrm{~m}$ & $1.53 \mathrm{~m}$ & $3.46 \mathrm{~m}$ & $2.94 \mathrm{~m}$ & & $6.26 \mathrm{~m}$ & & \\
& 3D & $1.86 \mathrm{~m}$ & $1.81 \mathrm{~m}$ & $4.46 \mathrm{~m}$ & $3.99 \mathrm{~m}$ & & $7.44 \mathrm{~m}$ & & 2.9 \\
Perth & $\mathrm{E}$ & $0.68 \mathrm{~m}$ & $1.26 \mathrm{~m}$ & $0.76 \mathrm{~m}$ & $1.39 \mathrm{~m}$ & $1.33 \mathrm{~m}$ & $1.49 \mathrm{~m}$ & $2.14 \mathrm{~m}$ & \\
(CUTA,NetR9) & $\mathrm{N}$ & $0.91 \mathrm{~m}$ & $1.35 \mathrm{~m}$ & $1.57 \mathrm{~m}$ & $1.57 \mathrm{~m}$ & $1.57 \mathrm{~m}$ & $2.02 \mathrm{~m}$ & $2.05 \mathrm{~m}$ & \\
& $\mathrm{U}$ & $2.06 \mathrm{~m}$ & $2.91 \mathrm{~m}$ & $2.70 \mathrm{~m}$ & $2.57 \mathrm{~m}$ & $3.30 \mathrm{~m}$ & $4.01 \mathrm{~m}$ & $3.96 \mathrm{~m}$ & \\
& 3D & $2.35 \mathrm{~m}$ & $3.44 \mathrm{~m}$ & $3.21 \mathrm{~m}$ & $3.31 \mathrm{~m}$ & $3.89 \mathrm{~m}$ & $4.73 \mathrm{~m}$ & $4.95 \mathrm{~m}$ & 2.3 \\
Chennai & $\mathrm{E}$ & $0.82 \mathrm{~m}$ & $1.02 \mathrm{~m}$ & $1.23 \mathrm{~m}$ & $1.44 \mathrm{~m}$ & $1.49 \mathrm{~m}$ & $1.56 \mathrm{~m}$ & $1.54 \mathrm{~m}$ & \\
(CHEN,NetR9) & $\mathrm{N}$ & $0.78 \mathrm{~m}$ & $0.76 \mathrm{~m}$ & $1.33 \mathrm{~m}$ & $1.76 \mathrm{~m}$ & $1.60 \mathrm{~m}$ & $1.29 \mathrm{~m}$ & $1.53 \mathrm{~m}$ & \\
& $\mathrm{U}$ & $2.62 \mathrm{~m}$ & $2.00 \mathrm{~m}$ & $1.81 \mathrm{~m}$ & $3.60 \mathrm{~m}$ & $3.48 \mathrm{~m}$ & $3.65 \mathrm{~m}$ & $4.66 \mathrm{~m}$ & \\
& 3D & $2.85 \mathrm{~m}$ & $2.37 \mathrm{~m}$ & $2.56 \mathrm{~m}$ & $4.25 \mathrm{~m}$ & $4.11 \mathrm{~m}$ & $4.17 \mathrm{~m}$ & $5.14 \mathrm{~m}$ & 2.2 \\
\hline
\end{tabular}

Overall, the results indicate a somewhat lower positioning performance for BeiDou as compared to GPS. Best results are generally obtained for the B1 single-frequency processing, for which a user equivalent range error (UERE) of 1.2-1.4 $\mathrm{m}$ (comprising both broadcast ephemeris errors and measurement errors) can be inferred from the ratio of the 3D RMS position errors and the position dilution of precision (PDOP). For dual-frequency solutions, UEREs of 1.9-2.6 $\mathrm{m}$ are obtained, which reflects the amplification of receiver noise and multipath when forming the ionosphere-free combination. Since an inferior positioning performance can also be observed for B2 and B3 single-frequency processing in comparison with B1-only solutions, remaining imperfections in the applied group delay parameters or inconsistent antenna phase centers may be suspected for these signals.

\section{SUMMARY AND CONCLUSIONS}

With the release of a public signal ICD in late Dec. 2012, worldwide users can now get access to the broadcast navigation messages transmitted by the BeiDou satellites and employ them for real-time navigation. The BeiDou navigation message exhibits a fair degree of communality with GPS (data rate, frame structure, orbital elements representations) but also differs in numerous details (parity algorithm, bit layout, orbit and ionosphere model). For the geostationary satellites of the BeiDou constellation a distinct navigation message type with a 10-times higher data rate is employed. This enables the transmission of differential correction data and a regional ionosphere map on top of the standard navigation data. BeiDou thus 
combines the services of a navigation satellite system with that of a satellite based augmentation system.

While the current ICD addresses only the single-frequency B1 navigation service, the BeiDou system can already be used for B2 and B3 as well as dual-frequency B1/B2 and B1/B3 positioning. Care must be taken, though, that BeiDou adopts a different concept for the handling of inter-signal biases than other navigation satellite systems and refers the broadcast clock offset to a single signal (B3) rather than an ionosphere-free combination. Users of the B2 frequency thus need to make use of a hidden group delay parameter in the navigation message or refer to differential code bias products derived from independent monitoring networks.

Based on comparison with satellite laser ranging data and post-processed orbit and clock products as well as single-point positioning results a signal-in-space range error of $1.2 \mathrm{~m}$ or better can be inferred for the BeiDou broadcast ephemeris. The value is considered as an upper limit of the actual navigation message accuracy since the comparison suffers from uncertainties in the antenna phase center and spacecraft attitude modelling. Also, the employed reference orbit and clock products are themselves of limited accuracy due to the low number of available monitoring stations. While the estimated SISRE is only moderately worse than the GPS constellation average of $0.8 \mathrm{~m}$, BeiDou users are also commonly affected by a less favourable position dilution of precision. For a set of five test sites in the AsiaPacific region (but outside the China mainland) representative single-point positioning accuracies of 2.5-5 m have been obtained in BeiDou-only solutions, while GPS-only solutions were typically accurate to 2-3.5 m. In both cases, ionospheric path delays have bee corrected through global ionosphere maps or eliminated through a dual-frequency combination.

With the given performance, BeiDou clearly lends itself as a standalone navigation system but can also favourably be combined with GPS to improve the overall integrity and robustness. Geodetic and surveying users can likewise benefit from the new constellation but an extensive characterization of spacecraft, antenna and signal properties is considered mandatory to a take full advantage of BeiDou in precise point positioning applications.

\section{ACKNOWLEDGEMENTS}

The authors are grateful to Xiaogong $\mathrm{Hu}$ (Shanghai Observatory) for valuable discussions concerning the interpretation and proper usage of the BeiDou navigation information. Satellite laser ranging observations of the BeiDou satellites have kindly been made available by the ILRS. Data from the Chennai station have kindly been made available by Trimble. The MultiGNSS Experiment (MGEX) is an initiative of the International GNSS Service (IGS). The contribution of all station providers and as well as the respective data and analysis centers is gratefully acknowledged.

\section{REFERENCES}

Beyerle G, Ramatschi M, Galas R, Schmidt T, Wickert J, Rothacher M (2009) A data archive of GPS navigation messages, GPS Solutions 13(1):35-41. DOI 10.1007/s10291-008-0095-y

CSNO (2012) BeiDou Navigation Satellite System Signal In Space Interface Control Document Open Service Signal B1I, Version 1.0, China Satellite Navigation Office, Dec 2012.

EU (2010) European GNSS (Galileo) Open Service Signal In Space Interface Control Document, OD SIS ICD, Issue 1.1, September 2010. 
Gao GX, Chan A, Lo S, De Lorenzo D, Todd Walter, Enge P (2009) Compass-M1 broadcast codes in E2, E5b, and E6 frequency bands, IEEE Journal of Selected Topics in Signal Processing 3(4):599-612. DOI 10.1109/JSTSP.2009.2025635

Gruber B (2012) GPS Program Update to ION GNSS 2012, Proc. ION GNSS 20912, Nashville TN.

Hauschild A, Montenbruck O, Sleewaegen JM, Huisman L, Teunissen P (2012a) Characterization of Compass M-1 Signals, GPS Solutions 16(1):117-126. DOI 10.1007/s10291-011-0210-3

Hauschild A, Montenbruck O, Steigenberger P (2012b) Short-Term Analysis of GNSS Clocks, GPS Solutions. DOI 10.1007/s10291-012-0278-4

He L, Ge M, Wang J, Wickert J, Schuh H (2013) Experimental Study on the Precise Orbit Determination of the BeiDou Navigation Satellite System, Sensors 13:2911-2928; doi:10.3390/s130302911

IGS RWG (2013) RINEX - The Receiver Independent Exchange Format, Version 3.02, International GNSS Service (IGS), RINEX Working Group and Radio Technical Commission for Maritime Services Special Committee 104 (RTCM-SC104), March 26, 2013.

Inaba N, Matsumoto A, Hase H, Kogure S, Sawabe M, Terada K (2009) Design Concept of Quasi Zenith Satellite System, Acta Astronautica 65(7-8):1068-1075, DOI 10.1016/j.actaastro.2009.03.068

IS-GPS-200 (2011) Navstar GPS Space Segment / Navigation User Segment Interfaces; Interface Specification IS-GPS-200, Revision F, 21 Sep. 2011, Global Positioning Systems Directorate.

Montenbruck O, Hauschild A, Steigenberger P, Hugentobler U, Riley S (2012) A COMPASS for Asia: First Experience with the BeiDou-2 Regional Navigation System, IGS Workshop 2012, 23-27 July 2012, Olsztyn, Poland.

Montenbruck O, Hauschild A (2013) Code Biases in Multi-GNSS Point Positioning, ION International Technical Meeting 2013, 28-30 Jan. 2013, San Diego.

Montenbruck O, Hauschild A, Steigenberger P, Hugentobler U, Teunissen P, Nakamura S (2013) Initial Assessment of the COMPASS/BeiDou-2 Regional Navigation Satellite System, GPS Solutions 17(2):211-222. DOI 10.1007/s10291-012-0272-x

Pearlman MR, Degnan JJ, Bosworth JM (2002) The International Laser Ranging Service, Advances in Space Research, 30(2):135-143. DOI:10.1016/S0273-1177(02)00277-6

Rizos Ch, Montenbruck O, Weber R, Weber G, Neilan R, Hugentobler U (2013) The IGS MGEX Experiment as a Milestone for a Comprehensive Multi-GNSS Service, ION Pacific PNT Meeting 2013, 22-25 April 2013, Honolulu.

Shi C, Zhao Q, Li M, Tang W, Hu Z, Lou Y, Zhang H, Niu X, Liu J (2012) Precise orbit determination of Beidou satellites with precise positioning, Science China Earth Sciences 55(7):1079-1086, DOI 10.1007/s11430-012-4446-8

Shi C, Zhao Q, Hu Z, Liu J (2013) Precise relative positioning using real tracking data from COMPASS GEO and IGSO satellites, GPS Solutions, 17(1):103-119. DOI 10.1007/s10291012-0264-x

Steigenberger P, Hugentobler U, Hauschild A, Montenbruck O (2013) Orbit and Clock Analysis of Compass GEO and IGSO Satellites, Journal of Geodesy. DOI 10.1007/s00190-013-0625-4

Warren DLM, Raquet JF (2003) Broadcast vs precise GPS ephemerides: a historical perspective, GPS Solutions 7(3):151-156. DOI 10.1007/s10291-003-0065-3

Wu X, Hu X, Wang G, Zhong H, Tang C (2013) Evaluation of COMPASS ionospheric model in GNSS positioning, Advances in Space Research 51:959-968. DOI 10.1016/j.asr.2012.09.039 